\title{
Identification and temporal expression of putative circadian clock transcripts in the amphipod crustacean Talitrus saltator
}

\author{
Joseph F O'Grady ${ }^{1}$ ， Laura S Hoelters ${ }^{1}$ ， Martin T Swain ${ }^{1}$ ， David C Wilcockson ${ }^{\text {Corresp. } 1}$ \\ ${ }^{1}$ Institute of Biological, Environmental and Rural Sciences, University of Wales, Aberystwyth, Aberystwyth, Ceredigion, SY23 3DA, United Kingdom \\ Corresponding Author: David C Wilcockson \\ Email address: dqw@aber.ac.uk
}

Background. Talitrus saltator is an amphipod crustacean that inhabits the supralittoral zone on sandy beaches in the Northeast Atlantic and Mediterranean. T. saltator exhibits endogenous locomotor activity rhythms and time-compensated sun and moon orientation, both of which necessitate at least one chronometric mechanism. Whilst their behaviour is well studied, currently there are no descriptions of the underlying molecular components of a biological clock in this animal, and very few in other crustacean species.

Methods. We harvested brain tissue from animals expressing robust circadian activity rhythms and used homology cloning and Illumina RNAseq approaches to sequence and identify the core circadian clock and clock-related genes in these samples. We assessed the temporal expression of these genes in timecourse samples from rhythmic animals using RNAseq.

Results. We identified a comprehensive suite of circadian clock gene homologues in $T$. saltator including the 'core' clock genes period (Talper), cryptochrome 2 (Talcry2), timeless (Taltim), clock (Talclk), and bmal1 (Talbmal1). In addition we describe the sequence and putative structures of 23 clock-associated genes including two unusual, extended isoforms of pigment dispersing hormone (PDH). We examined time-course RNAseq expression data, derived from tissues harvested from behaviourally rhythmic animals, to reveal rhythmic expression of these genes with approximately circadian period in Talper and Talbmal1. Of the clock-related genes, casein kinase $\| \beta$ (Talckllß), ebony (Talebony), jetlag (Taljetlag), pigment dispensing hormone (Talpdh), protein phosphatase 1 (Talpp1), shaggy (Talshaggy), sirt1 (Talsirt1), sirt7 (Talsirt7) and supernumerary limbs (Talslimb) show temporal changes in expression.

Discussion. We report the sequences of principle genes that comprise the circadian clock of $T$. saltator and highlight the conserved structural and functional domains of their deduced cognate proteins. Our sequencing data contribute to the growing inventory of described comparative clocks. Expression profiling of the identified clock genes illuminates tantalising targets, for experimental manipulation to elucidate the molecular and cellular control of clock-driven phenotypes in this crustacean. 
$1 \quad$ Identification and temporal expression of putative circadian

2 clock transcripts in the amphipod crustacean Talitrus saltator

3 Joseph F. O'Grady, Laura Hoelters, Martin Swain, David C. Wilcockson*

4 Institute of Biological, Environmental and Rural Sciences, Aberystwyth University, Penglais,

5 Aberystwyth, Ceredigion, Wales, SY23 3DA, UK

6 "Corresponding author email: $\underline{\text { dqw@aber.ac.uk }}$

7 Abstract

8 Background. Talitrus saltator is an amphipod crustacean that inhabits the supralittoral

9 zone on sandy beaches in the Northeast Atlantic and Mediterranean. T. saltator exhibits

10 endogenous locomotor activity rhythms and time-compensated sun and moon orientation,

11 both of which necessitate at least one chronometric mechanism. Whilst their behaviour is

12 well studied, currently there are no descriptions of the underlying molecular components of

13 a biological clock in this animal, and very few in other crustacean species.

14 Methods. We harvested brain tissues from animals expressing robust circadian activity

15 rhythms and used homology cloning and Illumina RNAseq approaches to sequence and

16 identify the core circadian clock and clock-related genes in these samples. We assessed the

17 temporal expression of these genes in time-course samples from rhythmic animals using

18 RNAseq.

19 Results. We identified a comprehensive suite of circadian clock gene homologues in $T$.

20 saltator including the 'core' clock genes period (Talper), cryptochrome 2 (Talcry2), timeless

21 (Taltim), clock (Talclk), and bmal1 (Talbmal1). In addition we describe the sequence and

22 putative structures of 23 clock-associated genes including two unusual, extended isoforms

23 of pigment dispersing hormone (PDH). We examined time-course RNAseq expression data,

24 derived from tissues harvested from behaviourally rhythmic animals, to reveal rhythmic

25 expression of Talper and Talbmal1 with a circadian period. Of the clock-related genes, casein 
26 kinase II $\beta$ (TalckII $\beta$ ), ebony (Talebony), jetlag (Taljetlag), pigment dispensing hormone

27 (Talpdh), protein phosphatase 1 (Talpp1), shaggy (Talshaggy), sirt1 (Talsirt1), sirt7 (Talsirt7)

28 and supernumerary limbs (Talslimb) show temporal changes in expression.

29 Discussion. We report the sequences of principle genes that comprise the circadian clock of

30 T. saltator and highlight the conserved structural and functional domains of their deduced

31 cognate proteins. Our sequencing data contribute to the growing inventory of described

32 comparative clocks. Expression profiling of the identified clock genes illuminates tantalising

33 targets, for experimental manipulation to elucidate the molecular and cellular control of

34 clock-driven phenotypes in this crustacean.

\section{Introduction}

36 Nearly all organisms are exposed to monotonous cyclic alterations in their environment.

37 Competence to gate behaviour and physiology in tune with these changes is orchestrated by

38 so-called circadian clocks, the cardinal features of which are: the clock mechanism is autonomous and endogenous i.e., it persists in the absence of external cues; the period of

40 the rhythm is temperature compensated and is entrained by relevant cyclic environmental

41 cues. For example, in the terrestrial realm, organisms invariably exhibit daily activity

42 rhythms in temporal correspondence to the light and dark of day and night. Our understanding of the molecular and cellular underpinnings of circadian clocks has advanced

44 tremendously in recent years and comparative studies have benefitted from the advent of next generation sequencing strategies to reveal clock genes in non-model species, including

46 crustaceans (Christie et al., 2013; De Pitta et al., 2013; Nesbit \& Christie 2014; Toullec et al., 47 2013). 
48 In the fruit fly brain about 150 cells, primarily in the protocerebrum and optic lobe,

49 contribute to the central clock oscillatory system, although this number varies throughout

50 arthropods. Indeed, in some Lepidoptera (Sauman et al., 2005) and Crustacea (Beckwith et

51 al., 2011; Zhang et al., 2013) this number seems to be much lower. The consensus arthropod

52 model of the central oscillatory mechanism is based on transcriptional/translational

53 feedback loops (TTFLs), with positive loops driving the expression of negative factors that

54 feed-back to inhibit the positive loops and hence their own transcription. Despite

55 interspecific variation, the principle mechanisms and components are conserved across

56 diverse taxa (For reviews see (Allada \& Chung 2010; Hardin 2009; Sandrelli et al., 2008)).

57 Briefly, in Drosophila the transcription factors CLOCK and CYCLE (CLK and CYC) drive the

58 expression of period (per) and timeless (tim). The translation of per and tim throughout the

59 evening and early night results in the cytoplasmic accumulation of their cognate proteins

60 that subsequently form stable heterodimers and translocate to the nucleus where they

61 interfere with the action of CLK and CYC to inhibit their own transcription. Under constant

62 conditions such as DD this autoregulatory feedback loop takes circa $24 \mathrm{~h}$ to complete due to

63 the opposing phosphorylation and dephosphorylation actions of various casein kinases and

64 phosphatases that alter circadian protein stability. For example, the stability of PER is

65 facilitated by casein kinase $1 \varepsilon(\mathrm{CK} 1 \varepsilon)$ or DOUBLETIME (DBT) phosphorylation and

66 translocation of the PER/TIM dimer is mediated by phosphorylation via glycogen synthase

67 kinase $\beta$ or "SHAGGY" (SGG). In fruit flies maintained under LD conditions the blue light

68 activated flavoprotein cryptochrome (dCRY1) acts as a transduction pathway to relay photic

69 stimuli to the TTFL. When photo-activated at dawn dCRY1 facilitates the degradation of

70 TIM, via the CRYPTOCHROME/JETLAG (JET) complex. This consequently leads to

71 destabilisation of PER, which is ubiquitinated by E3 ubiquitin ligase SUPERNUMERARY 
72 LIMBS (SLIMB), and degraded via the proteasome. The resultant de-repression of dCLOCK

73 and dCYCLE 'resets' the clock at dawn synchronising (or entraining) the system to the

74 ambient conditions. Protein phosphatases (PP) including PP2A and PP1 regulate these

75 phosphorylation events and so their action contributes to the temporal control of the

76 feedback loops. A secondary negative loop also governs precisely timed oscillations; the

77 CLK-CYC heterodimer initiates the transcription of the basic zipper (bZip) activator PAR

78 domain protein $1 \varepsilon$ (PDP1ع) and bZip transcriptional repressor vrille (vri) (Blau \& Young

79 1999) by binding E-boxes in the vri promoter. VRI accumulation culminates in the

80 repression of $c l k$ transcription by binding VRI/PAR domain protein $1 \varepsilon$ (PDP1ع) promoters

81 (Cyran et al., 2003). VRI dependent repression also mediates transcription of output mRNAs

82 that cycle in phase with clk, such as cryptochrome (Glossop et al., 2003). In mammals and

83 some arthropods CRYPTOCHROME 2 (CRY2) acts as a negative repressor of the CLK/BMAL

84 positive loop. In flies the actions of CLK/CYC E-boxes are also targeted by a basic-helix-loop-

85 helix repressor, CLOCKWORK ORANGE (CWO) which is thought to sustain rhythmicity

86 (Kadener et al., 2007; Lim et al., 2007; Matsumoto et al., 2007).

87 In insects the octadecapeptide pigment dispersing factor (PDF) that occurs in subsets of

88 clock neurons plays pivotal roles in synchronising oscillator neurons in the clock network.

89 Perturbation of PDH function leads to arrhythmicity or incompetence to adjust phase and

90 period of rhythmic phenotypes to changing environmental cues (Shafer \& Yao 2014). PDF,

91 or rather the orthologous pigment dispersing hormone (PDH) was first discovered in

92 crustaceans where they play a neurohormonal role as well as a neuromodulatory function,

93 effecting dispersion of retinal pigments and integumental chromatophores, including the

94 diurnal rhythm of this action. In crustaceans PDHs occur in several isoforms and the

95 neuroarchitecture of PDH cells is more complex than for PDF suggesting additional

96 functions to pigment dispersion. However, the defined role(s) for PDH in rhythmic 
97 phenotypes of crustaceans remains poorly described (Beckwith et al., 2011; Strauss $\mathcal{E}$

98 Dircksen 2010; Wilcockson et al., 2011)

99 Talitrus saltator lives in the sandy substratum of the supralittoral zone of beaches on the

100 European Atlantic coast and in the Mediterranean. During the day it remains buried in the

101 sand, emerging after dark to make foraging excursions along the land-beach axis. Before

102 dawn it relocates to its burrowing zone to seek refuge, reinterred in the sand. This circadian

103 locomotor activity persists in the absence of external cues and is thus endogenously driven

104 (Bregazzi \& Naylor 1972; Edwards \& Naylor 1987). Remarkably T. saltator also maintains its

105 preferred position on the shore by orientating using visual cues such as the sun and moon

106 as a compass guide (Pardi \& Papi 1952; Ugolini 2003; Ugolini et al., 1999; Ugolini 1999;

107 Ugolini et al., 2007). This capability necessitates compensation for azimuthal changes over

108 time (Ugolini et al., 2002). Thus, these clock-controlled phenotypes contribute to the fitness

109 of T. saltator.

110 The well-defined behavioural phenotypes of T. saltator enable a range of comparisons with

111 other organisms to be drawn. For example, another peracarid crustacean, the isopod

112 Eurydice pulchra possesses independent circatidal and circadian clocks (Zhang et al., 2013)

113 and the staggering time-compensated navigational mechanisms of the monarch butterfly

114 (Reppert 2006) are comparable to those of $T$. saltator. This brings the relevance of

115 comparative clock biology sharply into focus; detailed analysis of a diverse non-model

116 species may reveal commonalities or differences that give insight into how each has evolved

117 and functions. Thus T. saltator represents an excellent, tractable model for time-

118 compensated orientation.

119 Despite decades of elaborate behavioural analyses, the molecular basis of clock-driven

120 behavioural phenotypes in T. saltator is not understood, even at the most fundamental level. 
121 Therefore we sought to elucidate the sequences of canonical clock genes and their temporal

122 expression dynamics in T. saltator to provide a platform from which to explore the

123 neuromolecular mechanisms of circadian clock mediated phenotypes in this animal. This

124 work contributes also to the steadily growing inventory of crustacean transcriptomes and is

125 applicable to further exploration of comparative chronobiology and animal orientation in a

126 tractable and ecologically important model.

\section{Materials and methods}

128 Animal husbandry, tissue sampling and RNA extraction

129 Animals were caught by hand from Ynyslas beach, Wales, UK at night during their active

130 period and returned to glass tanks containing damp sand at ambient temperature $\left(17^{\circ} \mathrm{C}\right)$

131 and 12:12 LD regime and with lighting at dawn and dusk ramped/dimmed over 30 minutes

132 to approximate natural conditions. Animals were fed fish food flakes ad libitum. After 7 days

133 acclimation the rhythmic emergence and locomotor activity of a sub-sample of 60 animals

134 was established. T. saltator has been shown to express more robust and less variable

135 locomotor rhythms in small groups (Bregazzi \& Naylor 1972). Therefore, animals were

136 housed in groups of five in a glass tank containing 10cm-deep damp sand and

137 compartmentalized with Plexiglas dividers. Across each compartment infrared beams were

138 passed via bespoke recording apparatus fabricated by Trikinetics, Waltham, MA. All activity,

139 registered as interruptions to infrared beams was recorded via proprietary software on a

140 stand-alone PC. Activity data were recorded in one minute bins and was analysed and

141 plotted using ClockLab software (Actimetrics, Wilmette, IL) run via Matlab ${ }^{\circledR}$ v6.2. T. saltator

142 brains were rapidly dissected from animals drawn from the population shown to be

143 behaviourally rhythmic (see above). Dissections were done in ice-chilled DEPC treated

144 physiological saline. Ten brains were pooled to form each replicate at 3-hour intervals and

145 snap frozen in liquid nitrogen before storing at $-80^{\circ} \mathrm{C}$ until use. 
146 Total RNA was extracted using Trizol $^{\circledR}$ (Invitrogen ${ }^{\mathrm{Tm}}$, Thermo Fisher, UK) according to the

147 manufacturer's instructions except that an additional wash step in $75 \%$ ethanol of the RNA

148 pellet was introduced prior to drying and rehydration in $30 \mu \mathrm{DEPC}$-treated water. All RNA

149 samples were treated with Turbo DNA-free ${ }^{\mathrm{TM}}\left(\right.$ Ambion $^{\circledR}$, Thermo Fisher, UK) to remove

150 contaminating DNA. RNA was then pooled for degenerate PCR, RACE PCR and Illumina

151 HiSeq2500 sequencing, whilst RNA time-course samples were prepared separately for

152 temporal expression analysis.

\section{Degenerate PCR}

154 Initially we used a strategy of degenerate PCR and 5' and 3' Rapid amplification of cDNA ends

155 (RACE) to identify full-length core canonical clock genes. Full details of the PCR conditions used can

156 be found in File S1 and primers used are tabulated in Table S1.

157 Illumina RNAseq protocol

158 The TruSeq cDNA library preparation protocol (Illumina, Cambridge, UK) was carried out

159 on eight time-point samples according to manufacturer's instructions. Amplified cDNA was

160 run on a $1 \%$ agarose gel to validate correct library size range. The cDNA libraries were

161 sequenced on an Illumina RNA TruSeq sequencer (Illumina Inc.) and quality checked at

162 Aberystwyth University's Core Genomics Facility. Read library quality was investigated

163 using FastQC (http://www.bioinformatics.babraham.ac.uk/projects/fastqc/), thereafter all

164 samples were pooled and de novo transcriptome assembly was performed with Trinity

165 software, version 2012-10-05 (Grabherr et al., 2011). Transcripts smaller than 300 bases

166 were removed from the pooled assembly. This consisted of 186,495 contigs with a mean

167 length of $1215 \mathrm{bp}$ pairs and an assembly N50 statistic of $2371 \mathrm{bp}$. 
169 Using the Trinity downstream analysis tools, reads from each time-point were mapped to

170 the pooled assembly and transcript abundance estimation was carried out using RSEM (Li \&

171 Dewey 2011). In this way the FPKM (Fragments Per Kilobase of exon per Million fragments

172 mapped) values were calculated for each time point. Following this TPM values (transcripts

173 per million) were calculated following Wagner et al., (2012). Quantitative PCR methods for

174 TPM validation are given in File S1 and primers in Table S1. All data files for time-course

175 RNAseq can be found on the NCBI SRA data base, bioproject accession number 297565.

176 Transcriptome mining and sequence analysis

177 Assembled contigs were initially interrogated for canonical clock gene transcripts using the

178 programme BioEdit (Hall 1999), Basic local alignment (BLAST) searches

179 (http://blast.ncbi.nlm.nih.gov/Blast.cgi) were done using Drosophila melanogaster protein

180 sequences as search terms and tblastn search functions set with default parameters (e-value

181 threshold at $1 \mathrm{e}^{-100}$ except for $\mathrm{PDH}$, where stringency was relaxed to 1000$)$. Where no

182 sequence information was available for Drosophila (e.g. cryptochrome 2) alternative

183 arthropod sequences were used, details of which are given in Table 1. Contigs were

184 translated using EXPASY Translate tool (http://web.expasy.org/translate/) and the coding

185 sequences checked manually using blastP for homology to known circadian proteins.

186 EMBL SMART (Letunic et al., 2009) servers, using default parameters, were used to detect

187 and analyse the conserved functional domains and motifs of clock proteins. Deduced $T$.

188 saltator protein sequences were used to query the FlyBase (http://flybase.org) and NCBI

189 non-redundant protein databases using the blastp algorithm. The identity and similarity

190 between protein sequences were calculated using EMBOSS Pairwise Alignment Algorithms

191 (http://www.ebi.ac.uk). The server SignalP v.4 (Petersen et al., 2011) was used to predict

192 the presence and location of signal peptide cleavage sites.

193 Blast2GO analysis- transcriptome annotation 
194 Before running Blast2GO, the number of transcripts was further reduced by clustering them

195 using CD-HIT-EST (version 4.5.4) with default options except for the sequence identity

196 threshold, which was set to 95\% (Li \& Godzik 2006; Li et al., 2001). The clustering step

197 removes very similar splice variants, which may include some misassemblies, and it

198 significantly reduces the number of transcripts to be included in the time-consuming BLAST

199 analysis. In total 156,766 transcripts with a mean length of $1534 \mathrm{bp}$ and an assembly N50

200 statistic of $968 \mathrm{bp}$ were run through BLAST. Using the BLAST output the transcripts were

201 annotated using BLAST2GO software (Conesa et al., 2005; Gotz et al., 2008) including

202 reports for Gene Ontology (GO) terms and EC numbers for the KEGG pathway. The

203 BLAST2GO cut-off parameters used to filter out poor quality BLAST hits for the annotation

204 were as follows: Annotation rule cut-off $=55$; E-value $=1 \mathrm{e}-6$; Hit-HSP overlap $=0$; and the

205 GO weight $=5$..

206 Determining cycling transcripts

207 Rhythmicity in clock gene expression was determined using the JTK_CYCLE software 208 (http://openwetware.org/wiki/HughesLab:JTK_Cycle) developed by Professors Michael E.

209 Hughes, Karl Kornacker and John Hogenesch (Hughes et al., 2010; Miyazaki et al., 2011)

210 following the JTK_CYCLE Users guide. Changes in gene expression values (TPM) were also

211 analysed by one-way ANOVA.

212 Data availability

213 Raw data files have been deposited in public sequencing databases as indicated in the text

214 or at https://figshare.com/s/a2513243c63bf557b720.

215 Results

216 Animals entrained under 12:12LD regimes showed robust activity rhythms when released

217 into constant darkness (DD) with peak activity occurring in the middle of the subjective

218 night (Fig. 1A). Periodogram analysis of representative animals revealed a period (tau) of

$21924.15 \mathrm{~h}$ (Fig. 1B). The demonstration of rhythmicity in these animals was essential to our 
220 sampling strategy; tissue for gene discovery was taken across one complete daily cycle and

221 pooled to ensure capture of transiently expressed transcripts. Additionally, expression

222 profiling of transcriptome contigs required samples harvested from rhythmic animals in-

223 phase with respect to each other.

224 Identification of putative Talitrus saltator circadian proteins.

225 Our principle objective was to describe the cDNAs encoding the core elements of the

226 circadian clock system in T. saltator, initially adopting homology cloning and standard

227 sequencing approach but, subsequently superseded by RNAseq strategies. The Illumina

228 RNAseq generated 141,769,456 reads, 128,386,193 of these were assembled into clustered

229 156,766 contigs (minimum length 300bp). This T. saltator Transcriptome Shotgun Assembly

230 project has been deposited at DDBJ/EMBL/GenBank under the accession GDUJ00000000.

231 The version described in this paper is the first version, GDUJ01000000. From this

232 transcriptome we identified contigs encoding putative circadian clock genes (Table 1). The

233 coding regions and conserved functional domains of identified 'core' clock genes period

234 (Talper), timeless (Taltim), cryptochrome2 (Talcry2) and bmal1 (Talbmal1) and clock (Talclk)

235 are diagrammatically represented in Fig. 2. In the interests of space, alignments of all genes

236 (core and clock-related) are shown in Supplemental Figs S3-S8 and S11-S31 and all BLAST

237 results are tabulated in Tables S2-S4.

238 Cryptochrome 2

239 Degenerate PCR coupled with RACE PCR yielded an 1843 bp cDNA sequence coding for a

240565 amino acid protein; a putative cryptochrome 2 assigned TalCRY2. An identical sequence

241 was revealed in transcriptome data but assembled as two separate contigs (Table 1). High

242 fidelity PCR amplification and sequencing confirmed that Talcry2 is expressed as one

243 contiguous transcript. The deduced protein sequence contains two SMART identified

244 domains, a DNA photolyase domain and a FAD binding 7 domain (Figs. 2 and S1) and shared 
245 an identity of $46.4 \%$ and a similarity of $56.0 \%$ with Danaus plexippus CRY2 (Accession No.

246 ABA62409) (Zhu et al., 2005). The highest sequence identity protein in the NCBI non-

247 redundant database is that for the closely related isopod crustacean, Eurydice pulchra CRY2

248 sequence (Accession No. AGV28717; (Zhang et al., 2013).

249 Period and Timeless

250 For Talper we mined an 8001 bp contig encoding a putative full-length protein of 1557

251 amino acids aligning to dPER with an identity of $29 \%$ and a similarity of $50 \%$. The deduced

252 TalPER contains characteristic features of PER including two PAS domains and a PAC

253 domain as well as nuclear export signal, cytoplasmic localization nuclear localization signal

254 motifs. In common with the isopod E. pulchra, TalPER also has a CK1E binding site sequence

255 and Period C domain (Figs. 2 and S2). The putative TalPER sequence aligned most closely to

256 dPER-PB in FlyBase (FlyBase no. FBpp0304590) and in the NCBI database to Eurydice

257 pulchra PER (Accession no. AGV28714; (Zhang et al., 2013). We also used the Drosophila

258 melanogaster TIM (dTIM) protein (Accession No. AAC46920; (Myers et al., 1995) as a search

259 term to identify a 1209 bp contig with a coding region for a 402 amino acid protein sharing

260 sequence similarity to dTIM (identity of $24.3 \%$ and similarity of $41.0 \%$, Fig. S3). SMART

261 analysis of this sequence identified a 228 amino acid Timeless domain (Figs. 2 and S3). The

262 partial protein sequence, assigned TalTIM aligned most closely in the NCBI database to the

263 partial Timeless-like protein of the termite Zootermopsis nevadensis (Accession no.

264 KDR17447; (Terrapon et al.,, 2014) and in FlyBase to Timeout-PA (FlyBase No.

265 FBpp0082180).

266 Clock

267 Using the D. melanogaster CLK (dCLK) sequence (Accession No. AAC62234; (Bae et al., 268 1998) as search term, a single 5723 bp contig was identified as a candidate TalCLK encoding 
269 sequence. However, this sequence lacked a stop codon and we were unable to deduce the C-

270 terminal end of the protein, which is missing a C-terminal transactivation domain (Figs. 2

271 and S4). Nevertheless, our partial sequence contains SMART identified domains including

272 one PAS domain, one PAC domain and coiled coil domains, whilst missing a bHLH domain

273 and a second PAS domain present in dCLK. The TalCLK PAS domain is likely homologous to

274 the PAS-2 domain of dCLK as it aligned better (identity of $64.2 \%$ and a similarity of $80.6 \%$ )

275 than it does to the PAS-1 domain (identity of $17.3 \%$ and a similarity of $36.0 \%$ ). Moreover,

276 the PAC domains align closely with an identity of $75.0 \%$ and a similarity of $90.9 \%$ to that of

277 dCLK. The most significant hit for TalCLK in the NCBI database is CLK of the prawn

278 Macrobrachium rosenbergii (Accession No. AAX44045; (Yang et al., 2006). In FlyBase the

279 most similar protein is the D. melanogaster CLK orthologue (FlyBase No. FBpp0306710).

280 Cycle/Bmal1

281 By searching the transcriptome using the Drosophila melanogaster CYC (dCYC) sequence

282 (Accession No. AAF49107) (Adams et al., 2000), we identified two contigs as potential

283 TalCYC/BMAL encoding sequences each of which aligned closely to separate regions of dCYC

284 (Figs. 2 and S5). In fact these two contigs, when correctly oriented, overlap to form one

285 contiguous sequence encoding a characteristic bHLH domain, two PAS domains a PAC and a

286 C terminal transactivation domain. This sequence was confirmed by standard, Hi-fidelity

287 PCR, cloning and sequencing. TalCLK aligned with dCLK with an identity of $56 \%$ and

288 similarity of $77 \%$ and with $43 \%$ and $57 \%$ to BMAL1 of signal crayfish (Pacifasticus

289 leniusculus, Accession No. AFV39705). TalBMAL1 aligned with 44\% identity 58\% similarity

290 to E. pulchra BMAL1.

291 In addition to the 'core' clock elements, we also isolated a comprehensive suite of 'clock-

292 associated' transcripts from contigs that conceptually translate to proteins known to

293 contribute to circadian clock functioning (Table 1). In the interests of space we refer the 
294 reader to the Additional files; herein the majority of detail is omitted from the text.

295 Sequence alignments are displayed in Figs. S6-S26 whilst BLAST search outputs of identified

296 contig sequences to nucleotide, protein and FlyBase repositories are given in Tables S2-S4.

297 Casein Kinase II (CKII) $\alpha$ and $\beta$ subunits.

298 We identified single contigs coding for putative CK11 $\alpha$ and CKII $\beta$. For CK11 $\alpha$ we found a

2995147 bp contig enveloping a coding sequence for a 353 amino acid protein including a

300 highly conserved serine/threonine protein kinase catalytic domain (Table 1, Fig. S6). This

301 putative TalCKII $\alpha$ sequence aligned in the NCBI database most closely to human CK2 Chain

302 A (Accession no. 1NA7 A; (Niefind et al.,, 1998)) and CKII $\alpha$ from the copepod crustacean,

303 Paracyclopina nana (Accession no. AII16523; Kim et al.,, unpublished submission). For

304 CKII $\beta$ we identified a 1567 bp coding sequence for a 220 amino acid protein, including a

305 casein kinase regulatory subunit domain (Table 1, Fig. S6). This sequence aligned most

306 closely in the NCBI database to parasitic wood wasp Orussus abietinus CKIIß (Accession no.

307 XP_012287730).

308 Clockwork Orange (CWO)

309 A candidate $c w o$ contig was mined that encodes a partial protein sequence of 167 amino

310 acids (Table 1, Fig. S7) and included a SMART-identified bHLH domain and the Orange of

311 the Hairy/E (SPL) family domain. TalCWO matched most closely in the NCBI database to an

312 uncharacterized protein from the mite Metaseiulus occidentalis (Accession no. $\underline{\mathrm{XP}}$

313 003744690); however, the same sequence aligned with CLOCKWORK ORANGE from the

314 firebug Pyrrhocoris apterus (Accession no. AGI17571; (Bajgar et al., 2013) with an e value of $3158 \mathrm{e}^{-42}$.

316 Doubletime (DBT)

317 A putative $1092 \mathrm{bp} d b t$ contig coding for a deduced a full-length 310 amino acid protein was

318 identified containing a serine/threonine protein kinase catalytic domain (Table 1, Fig. S8). 
319 The deduced sequence aligned most closely in the NCBI database to DBT of the isopod

320 crustacean Eurydice pulchra (Accession no. AGV28719; (Zhang et al., 2013) and in FlyBase

321 to the Drosophila melanogaster orthologue DCO (FlyBase no. FBpp0306615).

322

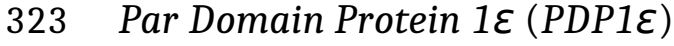

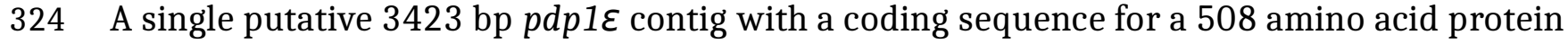

325 was identified containing a basic leucine zipper domain in the C-terminal region (Table 1,

326 Fig. S9). The deduced TalPDP1ع protein sequence aligned most closely in the NCBI database

327 to the hepatic leukaemia factor of the clonal raider ant Cerapachys biroi (Accession no.

328 EZA50108; (Oxley et al., 2014); however PDP1E from the mosquito Culex quinquefasciatus

329 (Accession no. XP 001865130; Atkinson et al.,, unpublished submission) is also a top

330 ranking BLAST hit aligned with an e value of $1 \mathrm{e}^{-35}$. In FlyBase the sequence aligned most

331 closely to Drosophila melanogaster PDP1 (FlyBase no. FBpp0289727).

332 Protein Phosphatase 1 (PP1)

333 A 1725bp contig was identified coding for a 357 putative PP1 containing a protein

334 phosphatase 2Ac catalytic domain of the serine/threonine phosphatase family (Table 1, Fig.

335 S10). The translated sequence aligned most closely to PP1 of the jumping ant Harpegnathos

336 saltator (Accession no. EFN86649; (Bonasio et al., 2010) in the NCBI database. In FlyBase

337 the sequence aligned most closely to Drosophila melanogaster PP1 (FlyBase no.

$338 \quad$ FBpp0306442).

339 Protein Phosphatase 2A (PP2A).

340 PP2A catalytic subunit "MICROTUBULE STAR" (MTS)

341 The Drosophila melanogaster MTS sequence (Accession No. AAF52567; (Adams et al., 2000),

342 dMTS) was used as a search term to identify a contig $2981 \mathrm{bp}$ in length and coding for a

343 putative 309 orthologue to dMTS with an identity of $91.9 \%$ and a similarity of $96.8 \%$

344 (Table 1, Fig. S11). The putative TalPP2A includes a protein phosphatase 2Ac catalytic

345 domain of the serine/threonine phosphatase family with an identity of $93.4 \%$ and a 
346 similarity of $98.2 \%$ to the fruit fly orthologue. The closest match in the NCBI database was

347 the putative serine/threonine protein phosphatase $\mathrm{PP}-\mathrm{V}$ from the body louse Pediculus

348 humanus corporis (Accession no. XP 002426726). The closest match in FlyBase was the

349 Drosophila melanogaster MTS homologue (FlyBase no. FBpp0310063).

350 PP2A catalytic subunit "WIDERBORST" (WBT)

351 We identified a $2474 \mathrm{bp}$ sequence that codes for a putative 458 amino acid protein. The

352 deduced protein sequence, although 66 amino acids shorter than that of Drosophila WBT

353 (the TalWBT protein lacks a sequence at the $C$ terminal end) aligned with the fruit fly

354 orthologue (identity of $73.8 \%$ ) \% (Table 1, Fig. S12). In common with other species the $T$.

355 saltator candidate contains B56 domains (serine/threonine-protein phosphatase 2A 56 kDa

356 regulatory subunit epsilon). The deduced TalWBT protein aligned most closely in the NCBI

357 database to serine/threonine-protein phosphatase 2A $56 \mathrm{kDa}$ regulatory subunit epsilon of

358 the red flour beetle Tribolium castaneum (Accession no. XP 971164) and in FlyBase to

359 Drosophila melanogaster WBT (FlyBase no. FBpp0084575).

360 PP2A catalytic subunit TWINS (TWS)

361 A unique $1633 \mathrm{bp}$ contig coding for a putative 445 amino acid protein with high identity to

362 Drosophila TWS, (Table 1, Fig. S13) was identified. The deduced sequence contained seven

363 WD40 domains identified using SMART. The protein aligned most closely in the NCBI

364 database to PP2A subunit B of the mud crab Scylla paramamosain (Accession no.

365 AFK24473; Liu and Ye, unpublished submission) and to Drosophila melanogaster TWINS

366 (FlyBase no. FBpp0081671).

367 Shaggy (SGG)

368 A 4413 bp transcript incorporating a coding region for a 418 amino acid protein that 369 contained a SMART identified serine/threonine protein kinase catalytic domain (Table 1,

370 Fig. S14). In the NCBI database the sequence aligned most closely to glycogen synthase 
371 kinase-3 of the turnip sawfly Athalia rosae (Accession no. XP 012256017) and in FlyBase to

372 Drosophila melanogaster SGG (FlyBase no. FBpp0070450).

373 Supernumerary Limbs (SLIMB)

374 A candidate 588 amino acid putative SLIMB protein encoded within a 2121 bp contig was

375 mined. This sequence included one D domain of beta-TrCP, one $\mathrm{F}$ box and seven WD40

376 domains (Table 1, Fig. S15). The deduced sequence aligned in the NCBI database most

377 closely to F-box/WD repeat-containing protein 1A of the termite Zootermopsis nevadensis

378 (Accession no. KDR19729; (Terrapon et al., 2014) and In the FlyBase to Drosophila

379 melanogaster SLIMB (FlyBase no. FBpp0303082).

380 Vrille (VRI)

381 A single 3949 bp contig incorporating coding sequence for a 509 amino acid protein was

382 identified as a putative vrille transcript. The putative TalVRI protein contains a SMART

383 identified basic region leucine zipper domain (Table 1, Fig. S16) and aligned most closely in

384 the NCBI database to Nuclear factor interleukin-3-regulated protein of the termite

385 Zootermopsis nevadensis (Accession no. KDR19729; (Terrapon et al., 2014) and in FlyBase to

386 Drosophila melanogaster VRI (FBpp0312171).

387 Ebony

388 A single $4380 \mathrm{bp}$ contig coding for a putative 974 amino acid protein with one AMP binding

389 domain, one AMP binding $\mathrm{C}$ domain and one PP binding domain was mined (Table1, Fig.

390 S17). The deduced protein most closely aligned in the NCBI database to the $\underline{\beta}$-alanyl

391 conjugating enzyme of the cockroach Periplaneta americana. P. americana EBONY

392 (Accession no. CAI26307; (Blenau \& Baumann 2005) has been shown to have $\beta$-alanyl-

393 dopamine (DA) synthase (BAS) enzymatic activity and the Drosophila melanogaster EBONY

394 sequence (Accession no. $\underline{\mathrm{ABO} 27280}$ ) aligned with the TalEBONY candidate sequence with

395 an e value of $8 \mathrm{e}^{-90}$, supported by EBONY (FlyBase no. FBpp0083505) being the most closely

396 aligned protein to the TalEBONY query in FlyBase. 
397 Pigment dispersing hormone 1 and II (PDHI and PDHII)

398 In the Trinity assembly, a 2430 bp contig was identified by searching for the conserved

399 NSELINS domain. The identified contig included a coding region for a putative 129 amino

400 acid TalPDH prepropeptide (TalPDH-I, Table 1, Fig. S18). This was subsequently extended to

4012471 bp by the addition of a 5' UTR by RACE PCR. The TalPDH-I contains a signal peptide

402 between residues 20-21, a 77 amino acid PDH-precursor-related peptide (PPRP) sequence

403 ending in a K-R dibasic cleavage site. Unusually however, the deduced mature peptide

404 sequence is 32 amino acids long and lacks an amidation signal. SMART identified PDH

405 domains are present in the TalPDH. The deduced 32 amino acid Tal-PDH-I mature peptide

406 has an identity of $40.6 \%$ and a similarity of $53.1 \%$ with the Uca pugilator $\beta-\mathrm{PDH}$ consensus

407 sequence.

408 A second putative TalPDH encoding transcript was found in the transcriptome of $3392 \mathrm{bp}$ in

409 length and including a coding region for an 89 amino acid prepropeptide that we assigned

410 TalPDH-II (Table 1, Fig. S18). This prepropeptide sequence was predicted to include a 23

411 residue signal peptide. The 43 amino acid PPRP thus extends from residue 24 to a K-R

412 dibasic cleavage site at residue 66 . The 23 residue mature peptide terminates in an

413 amidation signal and has an identity of $47.8 \%$ and a similarity of $69.6 \%$ with Uca pugilator

$414 \beta$-PDH consensus sequence. No domains were identified by SMART but a corresponding

415 PDH domain region was identified.

416 RORA

417 One 2217 bp partial contig, coding a 599 amino acid containing a C4 zinc finger domain was

418 identified (Table 1, Fig. S19) as a predicted candidate for a Talitrus homologue of the

419 hormone receptor RORA. The 3' HOLI ligand binding domain present in other RORA

420 proteins is not identified in TalRORA. It is possible that the HOLI ligand binding domain is

421 present in the unsequenced 3' section. The most closely aligned protein in the NCBI 
422 database is the house fly Musca domestica nuclear hormone receptor HR3 (Accession no.

423 XP 011290218). The Drosophila hormone receptor-like in 46 protein (FlyBase no.

$424 \quad$ FBpp0297438) was the closest protein to TalRORA in FlyBase.

425 Reverb

426 A $5385 \mathrm{bp}$ full length contig was identified, coding for a putative 1110 amino acid protein

427 containing both a C4 zinc finger domain and a HOLI ligand binding domain (Table 1, Fig.

428 S20). The sequence aligned most closely in the NCBI database to nuclear hormone receptor

429 E75, the non-mammalian REVERB homologue in the carpenter ant Camponotus floridanus

430 (Accession no. XP 011259848). The closest aligning protein in FlyBase was the Drosophila

431 melanogaster E75 protein (

432 Sirt 1, 2, 4, 6 and 7

433 Five contig sequences of lengths 4033 bp, 2275 bp, 2977 bp, 1209 bp and 5180 bp all

434 coding for partial proteins of length 955, 376, 354, 402 and 948 amino acids respectively

435 were identified in the transcriptome as homologues for SIRTUIN proteins 1, 2, 4, 6, and 7.

436 Each sequence contained one SMART identified SIR2 domain (Table 1, Figs. S21-S25).

437 TalSIRT1 aligned most closely to the trematode Schistosoma mansoni SIRT1 (Accession no.

438 ABG78545) in the NCBI database and Drosophila melanogaster SIRT1 (FBpp0080015) in

439 FlyBase. TalSIRT2 was most closely aligned to the red flour beetle Tribolium castaneum

440 hypothetical protein (Accession no. EFA06770) in the NCBI database and D. melanogaster

441 SIRT2 (FBpp0310647) in FlyBase. The putative TalSIRT4 protein is most closely aligned in

442 the NCBI database to SIRT4 of the Asian citrus psyllid Diaphorina citri (Accession no.

443 XP 008480918) and in FlyBase to the D. melanogaster SIRT4 (FBpp0070817). The TalSIRT6

444 sequence most closely aligns to the water flea Daphnia pulex hypothetical protein

445 (Accession no. EFX74386) in the NCBI database and in the FlyBase database to $D$.

446 melanogaster SIRT6 (FBpp0293897). TalSIRT7 most closely aligns in the NCBI database to 
447 the SIRT7 protein of the leafcutter bee Megachile rotundata (Accession no. XP 012143211)

448 and aligns most closely in the FlyBase database to the D. melanogaster SIRT7 protein

449 (FBpp0084733).

450 Jetlag

451 One 2454 bp contig coding a partial putative 458 amino acid protein was identified. This

452 candidate putative protein sequence contains one F-box domain and multiple leucine-rich

453 repeat domains (Table 1, Fig. S26). The sequence was most closely aligned in the NCBI

454 database to the red flour beetle Tribolium castaneum F-box/LRR-repeat protein (Accession

455 no. XP 008193983). In the FlyBase database the TalJET sequence aligned most closely to a $D$.

456 melanogaster protein from the F-box and leucine rich repeat region group (FBpp0111980).

457 Blast2Go analysis

458 Gene ontology (GO) annotations were categorised into the three groupings: 'Biological

459 Process', 'Molecular Function' and 'Cellular Component'. These categories of annotation

460 were most easily displayed graphically at ontology level 2 (Fig. 3) where the most frequent

461 Molecular Functions were "Binding" and "Catalytic activity"; the most common Biological

462 Processes were "Cellular and Metabolic"; and the most common Cellular Components were

463 “Cell Components" and "Organelle components". These GO term outputs were subsequently

464 compared with results from transcriptomes previously generated and analysed including

465 the larva of the moth Plutella xylostella (Xie et al., 2012), the silkworm Bombyx mori, the

466 parasitic nematode Teladorsagia circumcincta (Menon et al., 2012) and the North Atlantic

467 copepod Calanus finmarchicus (Lenz et al., 2014). Proportions of GO terms were all found to

468 be at broadly similar levels in all organisms transcriptomes and for each category analysed;

469 for example, in the category Molecular Function ontology level 2 'binding' terms for $T$.

470 saltator, P. xylostella, B. mori and T. circumcincta were $51 \%, 48 \%, 41 \%$ and $40 \%$ 
471 respectively whilst level 2 'catalytic activity' terms were $31 \%, 36 \%, 38 \%$ and $40 \%$ 472 respectively.

473 Temporal expression of clock genes

474 JTK_CYCLE analysis applied to TPM values indicated oscillations (limited to a period of 475 between $21-27 \mathrm{~h})$ in the core clock genes Talper $\left(q=1.69^{-07}\right)$ and Talbmal1 $(q=0.001)$ (Fig. 4).

476 Alternative analyses by ANOVA showed significant differences in mean values at each 477 interval for Talcry2 $\left(F_{7,30}=2.745, P=0.032\right)$, Talper $\left(F_{7,30}=4.86, P=0.002\right)$ and Talbmal1 $478\left(F_{7,30}=2.445, P=0.049\right)$. Of the clock-related genes Talpdh $\left(q=5.43^{-04}\right)$, TalckII $\beta(q=0.0017)$, 479 Talpp1 ( $\mathrm{q}=0.023)$, Talshaggy $(\mathrm{q}=0.021$, Talebony $(\mathrm{q}=0.028)$, Talsirt1 $(\mathrm{q}=0.001)$, Talsirt7 480 ( $q=0.037)$ and Taljetlag $(q=0.027)$ exhibited oscillatory expression profiles by JTK_CYCLE 481 analysis. Interrogation of these data by ANOVA showed TalCK11 $\beta\left(\mathrm{F}_{7,30}=3.284, P=0.014\right)$

482 Talpp1 $\left(F_{7,30}=3.99, P=0.005\right)$, Talsirt1 $\left(F_{7,30}=4.91, P=0.002\right)$ and Talslimb $\left(F_{7,30}=3.19, P=0.016\right)$ 483 to be differentially expressed over time. Talper mRNA accumulation increased 484 approximately 6-fold during early night and peaked towards the middle of the subjective 485 night (CT18-21) before diminishing at CT24 reaching a nadir at CT6. This profile is 486 approximately antiphasic to TalckII $\beta$, Talpp1, Talsirt1 and Talslimb that all showed peaks in 487 expected daytime. Talcry2 and Talbmal1 respective contigs also showed antiphasic 488 relationships to Talper. However, JTK_CYCLE analysis did not reveal significant rhythmicity 489 in the Talcry2 transcript.

490 Given the robust rhythm of Talper expression we chose this transcript with which to 491 validate our RNAseq expression data by Taqman quantitative PCR. RNA levels of Talper in 492 samples taken from an independent time-course experiment show peak expression $(\sim 3-$ 493 fold change from CT3) at CT19 $\left(F_{7,32}=4.54, \mathrm{P}=0.01\right.$, see Fig. S27). These data support those 
494 revealed by RNAseq with peak expression in the early to mid night albeit with a slightly

495 lower amplitude.

496 Reads for Talcwo were below levels acceptable for analysis and excluded from further

497 investigation.

498 Discussion

499 Talitrus saltator exhibits intriguing clock-driven behavioural phenotypes, including

500 circadian locomotor rhythms (Bregazzi \& Naylor 1972; Ugolini et al., 2007) and time-

501 compensated solar and lunar navigation (Ugolini et al., 1999; Ugolini 1999; Ugolini 2003;

502 Ugolini et al., 2007). Their abundance and suitability for behavioural experimentation make

503 them an excellent model for comparative clock analysis but to make the species more

504 genetically tractable we set out to define the neuromolecular components of its circadian

505 clock. Initially our strategy included homology cloning and RACE PCR to sequence full-

506 length Talcry2, including the 3' and 5' UTRs, but we subsequently exploited RNAseq

507 technologies to sequence the cerebral ganglia transcriptome and identify 'core' clock genes

508 and clock related genes, known in other species to play a role in normal clock functioning.

509 The quality of the Trinity assembled transcriptome was evaluated in a number of ways.

510 First, we set out to maximise coverage of temporally regulated transcripts by harvesting

511 brain tissue across a complete day-night cycle, and from behaviourally rhythmic animals.

512 The clock gene transcripts identified from our assemblies and the proportion of assembled

513 contigs for that encoded full-length proteins (17 out of 28) suggest we satisfied this aim.

514 Second, standard cloning and sequencing of several genes, including Talcry2, Talper and

515 Talbmal1 and Talpdh-I resulted in 100\% nucleotide alignment with the corresponding 
516 contig coding region. Third, confidence in the identity of our contigs as clock gene

517 transcripts was provided by the identification of conserved functional motifs in each

518 conceptual translation. This was reflected also in the BLAST searching and high confidence

519 levels of the search returns (expressed as e-values) from the NCBI nucleotide and protein 520 databases as well as FlyBase. Taken together, these attributes indicate that the

521 transcriptome is a faithful representation of all genes expressed in this tissue across a day522 night cycle. Furthermore, Blast2GO analysis of the assembled contigs resulted in broadly 523 similar numbers of GO terms and categorisation pattern to previous studies (Lenz et al., 524 2014; Menon et al., 2012; Xie et al., 2012). The few differences observed between GO term 525 numbers of T. saltator to other non-model species can be attributed to transcriptomes being 526 compared across different tissues of diverse species at different stages of development and 527 subjected to various environmental conditions.

528 Clock genes are well conserved across diverse taxa (Tauber \& Kyriacou 2005; Tauber et al., 529 2004) and the discovery of structurally similar transcripts in T. saltator was expected. 530 Nevertheless, our data yielded some interesting comparative insights. In T. saltator we failed 531 to find a CRY1 but the CRY2 showed very high sequence similarity to that of the isopod 532 Eurydice pulchra, also lacking a CRY1 but possessing a mammalian-like CRY2 that has been 533 demonstrated, in vivo to function as the principle negative repressor BMAL1:CLK 534 transcriptional activity (Zhang et al., 2013). Given the close relationship of T. saltator to E. 535 pulchra it is tempting to speculate that the CRY2 we describe is also a negative regulator in 536 the core oscillator system. 
537 Whilst Drosophila do not have a CRY2, other insects, such as the monarch butterfly, Danaus

538 plexippus have both CRY1 and CRY2 (Zhu et al., 2005) and is considered the ancestral state-

539 Cry2 being lost in fruit flies (Sandrelli et al., 2008). However, peracarid crustaceans, that

540 presumably predate insects, have only a CRY2, offering alternative evolutionary

541 perspectives on the central clock mechanism. However, the evolutionary status of

542 cryptochrome is likely highly complex; for example, in contrast to E. pulchra and T. saltator,

543 the copepod crustaceans, Calanus finmarchicus and Tigriopus californicus have been shown

544 to express a putative CRY1 transcript (Lenz et al., 2014; Nesbit \& Christie 2014) whilst some

545 insects such as the flour beetle, Tribolium castaneum and the honey bee, Apis mellifera have

546 only a mammalian CRY2 [10].

547 Using the Drosophila CYC as a search term we elucidated a CYC-like sequence in T. saltator 548 containing a bHLH and 2 PAS domains, but differing from Drosophila in that it also 549 contained a C-terminal transactivation domain, on the basis of which we assigned the $T$. 550 saltator sequence as a vertebrate-like BMAL1. A very similar structural scenario was

551 described for BMAL1 in E. pulchra and the water flea Daphnia pulex (Zhang et al 2013). In E.

552 pulchra deletion of the transactivation domain resulted in severely attenuated functionality

553 (of the EpBMAL:EpCLK dimer) in a cell-based in vitro luciferase assay (Zhang et al., 2013).

554 Again, whilst we have no experimental evidence to support the notion, the close structural

555 and phylogenetic relationship between T. saltator and E. pulchra hint at a similar role for 556 TalBMAL1.

557 Although our data reveal most of the clock gene candidates we had anticipated, some, such 558 as Taltim and Talclk were incomplete. However, given the presence of the conserved 
559 domains identified within the transcripts, we are confident that these partial sequences are

560 the products of the Taltim and Talclk genes in T. saltator. Manual cloning and sequencing

561 strategies is necessary to reveal the full-length sequences to these. We also uncovered two

562 unusual sequences identified as Talpdh. For TalPDH-I the N-terminal end of the mature

563 hormone contains the conserved NSE/ALINSSLLG signature but, the remaining sequence

564 extends beyond the expected 18 residues that define PDHs, including a further 14 residues,

565 and lacking an amidation signal. A second contig discovered with relaxed search stringency

566 also exhibited features of PDH-like peptides with a 23 amino acid signal peptide, a 43

567 residue PPRP and a 23 amino acid mature PDH with C-terminal amidation. Concentrated

568 efforts to locate other $\mathrm{PDH}$ candidates in our transcriptome were unsuccessful.

569 Interestingly, a combined transcriptomic and mass spectrometry approach on the

570 transcriptome/neuropeptidome of the krill Euphasia crystallorophias (Toullec et al., 2013)

571 revealed three $\mathrm{PDH}$ isoforms $\mathrm{PDH}-\mathrm{L} \beta 1, \mathrm{PDH}-\mathrm{L} \beta 2$ and $\mathrm{PDH}-\mathrm{L} \alpha$. In this species PDH-L $\beta 1$

572 expresses the characteristic $\mathrm{N}$-terminal 12 residues but has an extended C-terminus,

573 making the mature, amidated peptide 24 amino acids in length. The PDH-L2 $\beta$ is almost

574 identical except for $\operatorname{Ser}^{2}$ being substituted for $\mathrm{Ala}^{2}$, reminiscent of the case in E. pulchra

575 (Wilcockson et al., 2011). TalPDH-II also expresses the conserved N-terminus with a

576 conserved substitution of $\mathrm{Leu}^{4}$ for $\mathrm{Ile}^{4}$. In accord with the structure of PDH prepropeptides,

577 we observed the signal peptide and PPRP, separated from the mature peptide by a dibasic

578 cleavage site $(\mathrm{K}-\mathrm{R})$. These $\mathrm{PDH}$ preprohormone-like features and confirmation of the contig

579 sequences by standard and RACE PCR and cloning together with the unusual PDH isoforms

580 reported in euphausiids, allay our initial fears that our sequence data were mis-assemblies

581 or anomalies. The structural and functional significance of PDHs in T. saltator are worthy of

582 further investigation. 
583 Many organisms show cyclic changes in gene expression of core and clock-related genes as a

584 function of the TTFL. A feature of biological clocks is that they free-run in constant

585 conditions i.e. when divorced from environmental cues transcriptional/translational activity

586 of the central oscillator will persist with a circadian period in DD. We determined the

587 transcriptional dynamics of the T. saltator clock genes in behaviourally rhythmic animals by

588 mapping RNAseq sequencing reads for each time-course sample back to the assembled

589 transcriptome and analysing temporal changes in these data by JTK_CYCLE analysis and

590 ANOVA. Of the canonical clock genes only Talper and Talbmal were indicated as rhythmically

591 expressed by JTK_CYCLE analysis and ANOVA with Talcry 2 abundance varying in time

592 (ANOVA). In addition, the clock related genes, TalckII $\beta$, Talpp1, Talsirt1, Talsirt7 Talebony,

593 Taljetlag, Talshaggy and Talpdh and showed oscillating expression using the same analytical

594 parameters. TalCK11 $\beta$, Talpp1, Talsirt1 and Talslimb abundance differences were also noted

595 over time (ANOVA).

596 We chose the JTK_CYCLE algorithm because it is reported to be very robust to outliers and

597 returns low numbers of false-positives (Hughes et al., 2010). In our study we sacrificed an

598 extended sampling period for increased biological replicates and three-hour sampling

599 intervals. We are confident that this approach, coupled with the JTK_CYCLE data treatment

600 yielded genuinely cycling genes that offer suitable targets for further exploration.

601 We caution that, it would be entirely speculative to draw conclusions on the functional 602 significance of the rhythmicity or phase relationships of cyclic expression identified in the 603 current study. Although circadian genes and proteins are conserved and the central tenet of 604 the TTFL serves to describe the basis of rhythmicity across taxa, simple interspecific 
605 comparisons are confounded by the fact that clocks do show nuances in their organisation

606 of the TTFL, e.g. in E. pulchra only Eptim shows robust cycling in animals expressing 607 circadian and circatidal behaviour and yet Epper, which is non-rhythmically expressed, 608 appears essential to circadian phenotypes (Zhang et al., 2013). Indeed, rhythmicity in gene 609 expression itself is not a prerequisite for a functional clock (Lakin-Thomas 2006). The 610 tremendous scope for complex interplay of gene products and their cognate proteins will 611 only be revealed by more targeted experimental approaches in non-model species. 612 Nevertheless, our data indicate that sub-sets of clock and clock-related genes in T. saltator 613 continue to cycle in free-running conditions and thus represent tantalising targets for 614 investigation to uncover the role of these genes in either circadian or circalunidian (24.8h) 615 rhythms. For example, T. saltator has the capability to orientate by the sun and moon 616 suggesting they may have separate daily and lunidian clocks (Ugolini et al., 1999). Indeed a 617 precedence for independent clock mechanisms in marine organisms has been set, e.g., E. 618 pulchra has been shown to possess a dual clock system with separate clocks orchestrating 619 circatidal and circadian phenotypes and the marine worm Platyneries dumerilli employs 620 separate mechanisms to keep circadian as well as lunar time (Zantke et al., 2013). The 621 sequence data and expression analysis described here provide a foot-hold in a behaviourally 622 tractable system from which we can address these questions.

623 In conclusion we have sequenced the entire brain transcriptome of an ecologically 624 important beach crustacean that exhibits intriguing clock-controlled phenotypes and report 625 the sequences of key clock and clock-related genes that are likely key players in these 626 phenotypes. Our dataset is one of only very few to describe the putative clock mechanism in 
627 a non-model crustacean and contribute to the growing inventory of crustacean and non-

628 model transcriptomes that may have wide-ranging utility in the research community.

\section{Acknowledgements}

630 Thanks to Justin Pachebat, (Aberystwyth University) for his advice and help with initial

631 transcriptome sequencing strategies.

632

633

634

635

636

637

638

639

640

641

642

643

644

645

646

647

648

649

650

651

652

653

654

655

656

657

658

\section{References}

Adams MD, Celniker SE, Holt RA, Evans CA, Gocayne JD, Amanatides PG, Scherer SE, Li PW, Hoskins RA, Galle RF, George RA, Lewis SE, Richards S, Ashburner M, Henderson SN, Sutton GG, Wortman JR, Yandell MD, Zhang Q, Chen LX, Brandon RC, Rogers YH, Blazej RG, Champe M, Pfeiffer BD, Wan KH, Doyle C, Baxter EG, Helt G, Nelson CR, Gabor GL, Abril JF, Agbayani A, An HJ, Andrews-Pfannkoch C, Baldwin D, Ballew RM, Basu A, Baxendale J, Bayraktaroglu L, Beasley EM, Beeson KY, Benos PV, Berman BP, Bhandari D, Bolshakov S, Borkova D, Botchan MR, Bouck J, Brokstein P, Brottier P, Burtis KC, Busam DA, Butler H, Cadieu E, Center A, Chandra I, Cherry JM, Cawley S, Dahlke C, Davenport LB, Davies P, de Pablos B, Delcher A, Deng Z, Mays AD, Dew I, Dietz SM, Dodson K, Doup LE, Downes M, Dugan-Rocha S, Dunkov BC, Dunn P, Durbin KJ, Evangelista CC, Ferraz C, Ferriera S, Fleischmann W, Fosler C, Gabrielian AE, Garg NS, Gelbart WM, Glasser K, Glodek A, Gong F, Gorrell JH, Gu Z, Guan P, Harris M, Harris NL, Harvey D, Heiman TJ, Hernandez JR, Houck J, Hostin D, Houston KA, Howland TJ, Wei MH, Ibegwam C, Jalali M, Kalush F, Karpen GH, Ke Z, Kennison JA, Ketchum KA, Kimmel BE, Kodira CD, Kraft C, Kravitz S, Kulp D, Lai Z, Lasko P, Lei Y, Levitsky AA, Li J, Li Z, Liang Y, Lin X, Liu X, Mattei B, McIntosh TC, McLeod MP, McPherson D, Merkulov G, Milshina NV, Mobarry C, Morris J, Moshrefi A, Mount SM, Moy M, Murphy B, Murphy L, Muzny DM, Nelson DL, Nelson DR, Nelson KA, Nixon K, Nusskern DR, Pacleb JM, Palazzolo M, Pittman GS, Pan S, Pollard J, Puri V, Reese MG, Reinert K, Remington K, Saunders RD, Scheeler F, Shen H, Shue BC, Siden-Kiamos I, Simpson M, Skupski MP, Smith T, Spier E, Spradling AC, Stapleton M, Strong R, Sun E, Svirskas R, Tector C, Turner R, Venter E, Wang AH, Wang X, Wang ZY, Wassarman DA, Weinstock GM, Weissenbach J, Williams SM, WoodageT, Worley KC, Wu D, Yang S, Yao QA, Ye J, Yeh RF, Zaveri JS, Zhan M, Zhang G, Zhao Q, Zheng L, Zheng XH, Zhong FN, Zhong W, Zhou X, Zhu S, Zhu X, Smith HO, Gibbs RA, Myers EW, Rubin GM, and Venter JC. 2000. The genome sequence of Drosophila melanogaster. Science 287:2185-2195. Allada R, and Chung BY. 2010. Circadian organization of behavior and physiology in Drosophila. Annu Rev Physiol 72:605-624. 10.1146/annurev-physiol-021909-135815 [doi] 
659

660

661

662

663

664

665

666

667

668

669

670

671

672

673

674

675

676

677

678

679

680

681

682

683

684

685

686

687

688

689

690

691

692

693

694

695

696

697

698

699

700

701

702

703

704

705

706

707

708

709
Bae K, Lee C, Sidote D, Chuang KY, and Edery I. 1998. Circadian regulation of a Drosophila homolog of the mammalian Clock gene: PER and TIM function as positive regulators. Mol Cell Biol 18:6142-6151.

Bajgar A, Jindra M, and Dolezel D. 2013. Autonomous regulation of the insect gut by circadian genes acting downstream of juvenile hormone signaling. Proc Natl Acad Sci U S A 110:4416-4421. $10.1073 /$ pnas. 1217060110

Beckwith EJ, Lelito KR, Hsu YW, Medina BM, Shafer O, Ceriani MF, and de la Iglesia HO. 2011. Functional Conservation of Clock Output Signaling between Flies and Intertidal Crabs. J Biol Rhythms 26:518-529. 26/6/518 [pii]10.1177/0748730411420242 [doi]

Blau J, and Young MW. 1999. Cycling vrille expression is required for a functional Drosophila clock. Cell 99:661-671.

Blenau W, and Baumann A. 2005. Molecular characterization of the ebony gene from the American cockroach, Periplaneta americana. Arch Insect Biochem Physiol 59:184-195.

10.1002/arch.20064

Bonasio R, Zhang G, Ye C, Mutti NS, Fang X, Qin N, Donahue G, Yang P, Li Q, Li C, Zhang P, Huang Z, Berger SL, Reinberg D, Wang J, and Liebig J. 2010. Genomic comparison of the ants Camponotus floridanus and Harpegnathos saltator. Science 329:1068-1071. $10.1126 /$ science. 1192428

Bregazzi PK, and Naylor E. 1972. Locomotor activity rythm of Talitrus saltator (Montagu) (Crustacean, Amphipoda). J Exp Biol 57:375-391.

Christie AE, Fontanilla TM, Nesbit KT, and Lenz PH. 2013. Prediction of the protein components of a putative Calanus finmarchicus (Crustacea, Copepoda) circadian signaling system using a de novo assembled transcriptome. Comp Biochem Physiol D-Genomics \& Proteomics 8:165-193. 10.1016/j.cbd.2013.04.002

Conesa A, Gotz S, Garcia-Gomez JM, Terol J, Talon M, and Robles M. 2005. Blast2GO: a universal tool for annotation, visualization and analysis in functional genomics research. Bioinformatics 21:3674-3676. 10.1093/bioinformatics/bti610

Cyran SA, Buchsbaum AM, Reddy KL, Lin MC, Glossop NR, Hardin PE, Young MW, Storti RV, and Blau J. 2003. vrille, Pdp1, and dClock form a second feedback loop in the Drosophila circadian clock. Cell 112:329-341.

De Pitta C, Biscontin A, Albiero A, Sales G, Millino C, Mazzotta GM, Bertolucci C, and Costa R. 2013. The Antarctic Krill Euphausia superba Shows Diurnal Cycles of Transcription under Natural Conditions. PLoS One 8. ARTN e6865210.1371/journal.pone.0068652

Edwards JM, and Naylor E. 1987. Endogenous circadian changes in orientational behavior of Talitrus saltator. J Mar Biol Assoc UK 67:17-26.

Glossop NR, Houl JH, Zheng H, Ng FS, Dudek SM, and Hardin PE. 2003. VRILLE feeds back to control circadian transcription of Clock in the Drosophila circadian oscillator. Neuron 37:249-261.

Gotz S, Garcia-Gomez JM, Terol J, Williams TD, Nagaraj SH, Nueda MJ, Robles M, Talon M, Dopazo J, and Conesa A. 2008. High-throughput functional annotation and data mining with the Blast2GO suite. Nucleic Acids Res 36:3420-3435. 10.1093/nar/gkn176

Grabherr MG, Haas BJ, Yassour M, Levin JZ, Thompson DA, Amit I, Adiconis X, Fan L, Raychowdhury R, Zeng Q, Chen Z, Mauceli E, Hacohen N, Gnirke A, Rhind N, di Palma F, Birren BW, Nusbaum C, Lindblad-Toh K, Friedman N, and Regev A. 2011. Full-length transcriptome assembly from RNA-Seq data without a reference genome. Nat Biotechnol 29:644-652. 10.1038/nbt.1883

Hall TA. 1999. BioEdit: a user friendly biological sequenc alignment editor and analysis program for Windows 95/98/NT. Nucleic Acid Symposium. p 95-98.

Hardin PE. 2009. Molecular mechanisms of circadian timekeeping in Drosophila. Sleep and Biological Rhythms 7:235-242. 10.1111/j.1479-8425.2009.00412.x

Hughes ME, Hogenesch JB, and Kornacker K. 2010. JTK_CYCLE: an efficient nonparametric algorithm for detecting rhythmic components in genome-scale data sets. J Biol Rhythms 25:372-380. $10.1177 / 0748730410379711$ 
710

711

712

713

714

715

716

717

718

719

720

721

722

723

724

725

726

727

728

729

730

731

732

733

734

735

736

737

738

739

740

741

742

743

744

745

746

747

748

749

750

751

752

753

754

755

756

757

758

759

760

761
Kadener S, Stoleru D, McDonald M, Nawathean P, and Rosbash M. 2007. Clockwork Orange is a transcriptional repressor and a new Drosophila circadian pacemaker component. Genes Dev 21:1675-1686. 10.1101/gad.1552607

Lakin-Thomas PL. 2006. Transcriptional feedback oscillators: maybe, maybe not. J Biol Rhythms 21:83-92. 10.1177/0748730405286102

Lenz PH, Roncalli V, Hassett RP, Wu LS, Cieslak MC, Hartline DK, and Christie AE. 2014. De Novo Assembly of a transcriptome for Calanus finmarchicus (Crustacea, Copepoda) - the dominant zooplankter of the North Atlantic Ocean. PLoS One 9. ARTN e8858910.1371/journal.pone.0088589

Letunic I, Doerks T, and Bork P. 2009. SMART 6: recent updates and new developments. Nucleic Acids Res 37:D229-D232. 10.1093/nar/gkn808

Li B, and Dewey CN. 2011. RSEM: accurate transcript quantification from RNA-Seq data with or without a reference genome. BMC Bioinformatics 12:323. 10.1186/1471-2105-12-323

Li W, and Godzik A. 2006. Cd-hit: a fast program for clustering and comparing large sets of protein or nucleotide sequences. Bioinformatics 22:1658-1659. 10.1093/bioinformatics/btl158

Li W, Jaroszewski L, and Godzik A. 2001. Clustering of highly homologous sequences to reduce the size of large protein databases. Bioinformatics 17:282-283.

Lim C, Chung BY, Pitman JL, McGill JJ, Pradhan S, Lee J, Keegan KP, Choe J, and Allada R. 2007. Clockwork orange encodes a transcriptional repressor important for circadian-clock amplitude in Drosophila. Curr Biol 17:1082-1089. S0960-9822(07)01418-2 [pii]10.1016/j.cub.2007.05.039 [doi]

Matsumoto A, Ukai-Tadenuma M, Yamada RG, Houl J, Uno KD, Kasukawa T, Dauwalder B, Itoh TQ, Takahashi K, Ueda R, Hardin PE, Tanimura T, and Ueda HR. 2007. A functional genomics strategy reveals clockwork orange as a transcriptional regulator in the Drosophila circadian clock. Genes Dev 21:1687-1700. 10.1101/gad.1552207

Menon R, Gasser RB, Mitreva M, and Ranganathan S. 2012. An analysis of the transcriptome of Teladorsagia circumcincta: its biological and biotechnological implications. BMC Genomics 13. Artn S1010.1186/1471-2164-13-S7-S10

Miyazaki M, Schroder E, Edelmann SE, Hughes ME, Kornacker K, Balke CW, and Esser KA. 2011. Ageassociated disruption of molecular clock expression in skeletal muscle of the spontaneously hypertensive rat. PLoS One 6:e27168. 10.1371/journal.pone.0027168

Myers MP, Wagersmith K, Wesley CS, Young MW, and Sehgal A. 1995. Positional cloning and sequence analysis of the Drosophila Clock gene, Timeless. Science 270:805-808. DOI $10.1126 /$ science.270.5237.805

Nesbit KT, and Christie AE. 2014. Identification of the molecular components of a Tigriopus californicus (Crustacea, Copepoda) circadian clock. Comp Biochem Physiology D-Genomics $\&$ Proteomics 12:16-44. 10.1016/j.cbd.2014.09.002

Oxley PR, Ji L, Fetter-Pruneda I, McKenzie SK, Li C, Hu HF, Zhang GJ, and Kronauer DJC. 2014. The Genome of the Clonal Raider Ant Cerapachys biroi. Curr Biol 24:451-458. 10.1016/j.cub.2014.01.018

Pardi L, and Papi F. 1952. Die Sonne Als kompass bei Talitrus saltator (Montagu), (Amphipoda, Talitridae). Naturwissenschaften 39:262-263.

Petersen TN, Brunak S, von Heijne G, and Nielsen H. 2011. SignalP 4.0: discriminating signal peptides from transmembrane regions. Nat Methods 8:785-786. 10.1038/nmeth.1701

Reppert SM. 2006. A colorful model of the circadian clock. Cell 124:233-236. 10.1016/j.cell.2006.01.009

Sandrelli F, Costa R, Kyriacou CP, and Rosato E. 2008. Comparative analysis of circadian clock genes in insects. Insect Mol Biol 17:447-463. IMB832 [pii] 10.1111/j.1365-2583.2008.00832.x [doi]

Sauman I, Briscoe AD, Zhu H, Shi D, Froy O, Stalleicken J, Yuan Q, Casselman A, and Reppert SM. 2005. Connecting the navigational clock to sun compass input in monarch butterfly brain. Neuron 46:457-467. 10.1016/j.neuron.2005.03.014 
762

763

764

765

766

767

768

769

770

771

772

773

774

775

776

777

778

779

780

781

782

783

784

785

786

787

788

789

790

791

792

793

794

795

796

797

798

799

800

801

802

803

804

805

806

807

808

809

810

811

812

813
Shafer OT, and Yao Z. 2014. Pigment-dispersing factor signaling and circadian rhythms in insect locomotor activity. Curr Opin Insect Sci 1:73-80. 10.1016/j.cois.2014.05.002

Strauss J, and Dircksen H. 2010. Circadian clocks in crustaceans: identified neuronal and cellular systems. Front Biosci (Landmark Ed) 15:1040-1074.

Tauber E, and Kyriacou CP. 2005. Molecular evolution and population genetics of circadian clock genes. Methods Enzymol 393:797-817. S0076687905930425 [pii]10.1016/S00766879(05)93042-5 [doi]

Tauber E, Last KS, Olive PJ, and Kyriacou CP. 2004. Clock gene evolution and functional divergence. $J$ Biol Rhythms 19:445-458.

Terrapon N, Li C, Robertson HM, Ji L, Meng XH, Booth W, Chen ZS, Childers CP, Glastad KM, Gokhale K, Gowin J, Gronenberg W, Hermansen RA, Hu HF, Hunt BG, Huylmans AK, Khalil SMS, Mitchell RD, Munoz-Torres MC, Mustard JA, Pan HL, Reese JT, Scharf ME, Sun FM, Vogel H, Xiao J, Yang W, Yang ZK, Yang ZQ, Zhou JJ, Zhu JW, Brent CS, Elsik CG, Goodisman MAD, Liberles DA, Roe RM, Vargo EL, Vilcinskas A, Wang J, Bornberg-Bauer E, Korb J, Zhang GJ, and Liebig J. 2014. Molecular traces of alternative social organization in a termite genome. Nat Communications 5. ARTN 3636 10.1038/ncomms4636

Toullec JY, Corre E, Bernay B, Thorne MA, Cascella K, Ollivaux C, Henry J, and Clark MS. 2013. Transcriptome and peptidome characterisation of the main neuropeptides and peptidic hormones of a euphausiid: the Ice Krill, Euphausia crystallorophias. PLoS One 8:e71609. 10.1371/journal.pone.0071609

Ugolini A. 2003. Activity rhythms and orientation in sandhoppers (Crustacea, Amphipoda). Front Biosci 8:s722-732.

Ugolini A, Melis C, Innocenti R, Tiribilli B, and Castellini C. 1999. Moon and sun compasses in sandhoppers rely on two separate chronometric mechanisms. Proc Roy Soc B-Biological Sciences 266:749-752.

Ugolini A, Melis, and Innocenti, R. 1999. Moon orientation in adult and young sandhoppers. J Comp Physiol A-Sensory Neural and Behavioral Physiology 184:9-12.

Ugolini A, Somigli S, Pasquali V, and Renzi P. 2007. Locomotor activity rhythm and sun compass orientation in the sandhopper Talitrus saltator are related. J Comp Physiol A-Sensory Neural and Behavioral Physiology 193:1259-1263. 10.1007/s00359-007-0277-z

Ugolini A, Tiribilli B, and Boddi V. 2002. The sun compass of the sandhopper Talitrus saltator: the speed of the chronometric mechanism depends on the hours of light. J Exp Biol 205:32253230.

Wagner GP, Kin K, and Lynch VJ. 2012. Measurement of mRNA abundance using RNA-seq data: RPKM measure is inconsistent among samples. Theory Biosci 131:281-285. 10.1007/s12064-012-0162-3

Wilcockson DC, Zhang L, Hastings MH, Kyriacou CP, and Webster SG. 2011. A novel form of pigmentdispersing hormone in the central nervous system of the intertidal marine isopod, Eurydice pulchra (leach). J Comp Neurol 519:562-575. 10.1002/cne.22533 [doi]

Xie W, Lei YY, Fu W, Yang ZX, Zhu X, Guo ZJ, Wu QJ, Wang SL, Xu BY, Zhou XG, and Zhang YJ. 2012. Tissue specific transcriptome profiling of Plutella Xylostella third Instar larval midgut. Int J Biol Sci 8:1142-1155. 10.7150/ijbs.4588

Yang JS, Dai ZM, Yang F, and Yang WJ. 2006. Molecular cloning of Clock cDNA from the prawn, Macrobrachium rosenbergii. Brain Res 1067:13-24. 10.1016/j.brainres.2005.10.003

Zantke J, Ishikawa-Fujiwara T, Arboleda E, Lohs C, Schipany K, Hallay N, Straw AD, Todo T, and Tessmar-Raible K. 2013. Circadian and circalunar clock interactions in a marine annelid. Cell Rep 5:99-113. 10.1016/j.celrep.2013.08.031

Zhang L, Hastings MH, Green EW, Tauber E, Sladek M, Webster SG, Kyriacou CP, and Wilcockson DC. 2013. Dissociation of circadian and circatidal timekeeping in the marine crustacean Eurydice pulchra. Curr Biol 23:1863-1873. 10.1016/j.cub.2013.08.038

Zhu H, Yuan Q, Briscoe AD, Froy O, Casselman A, and Reppert SM. 2005. The two CRYs of the butterfly. Curr Biol 15:R953-954. 10.1016/j.cub.2005.11.030 
Figure 1

Talitrus saltator exhibits free-running circadian locomotor activity rhythms

A. Plots of activity in five representative animals removed from the shore and held in DD over 9 days. Grey and black bars show time of subjective night and day, respectively. B. Chi Square Periodogram analysis of activity data of five representative animals recorded in DD over 9 days. The period of activity $(\tau)$ is shown inside the plot. Red line represents significance at $\mathrm{P}<0.001$. 
A

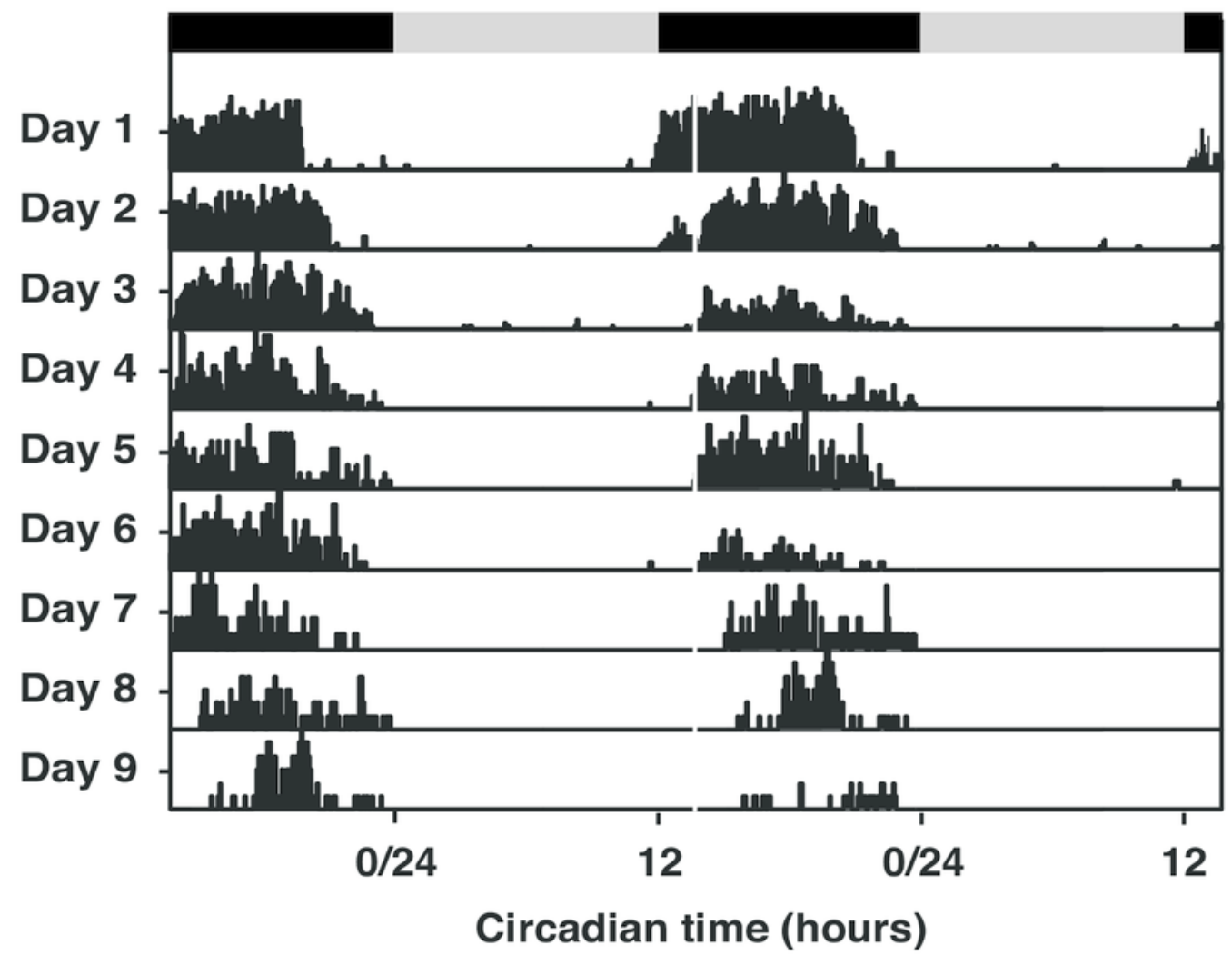

B

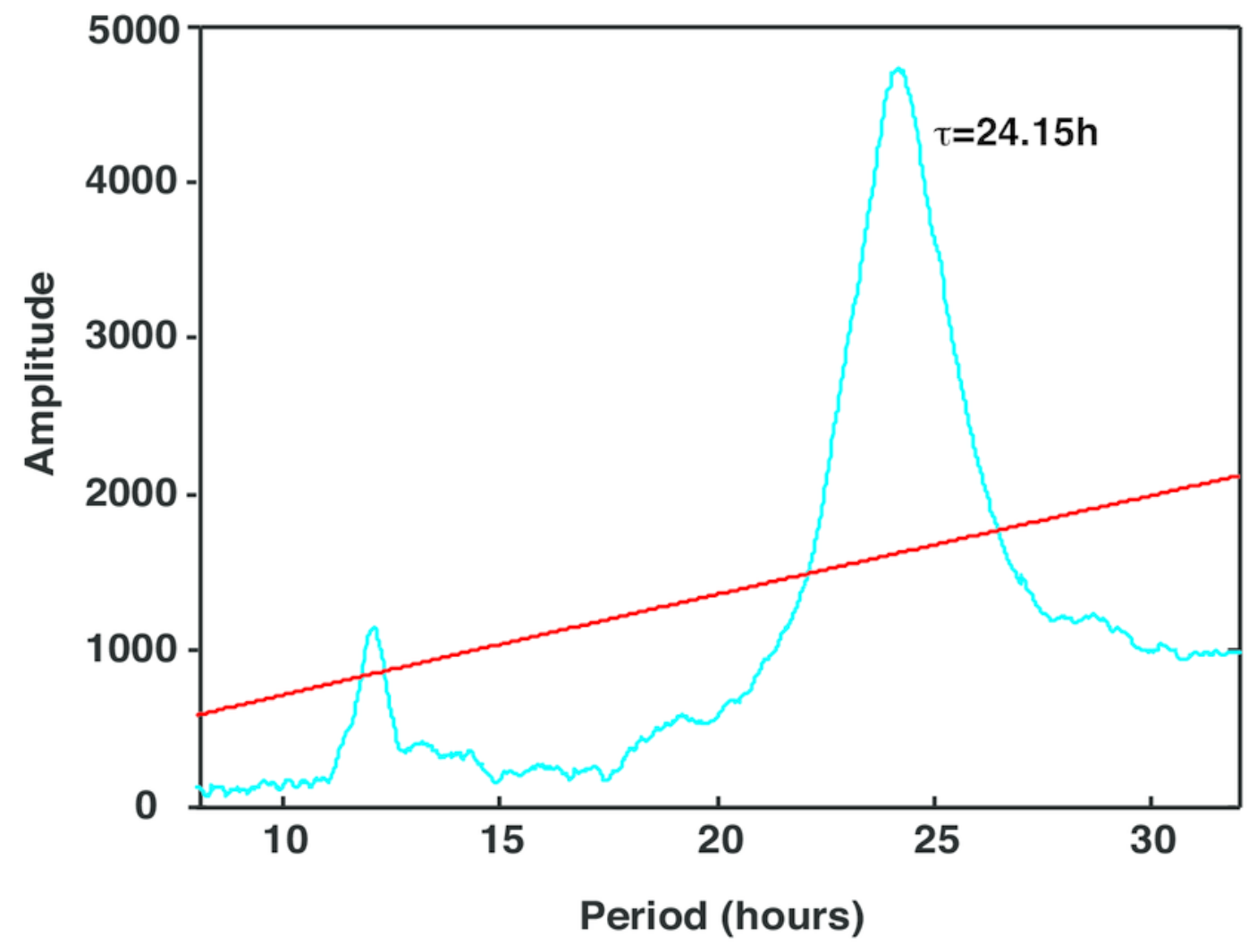


Figure 2

Cartoon representing conserved domains of core clock gene proteins

Putative Talitrus core clock protein amino acid sequences aligned with other arthropod circadian clock protein sequences. SMART identified domains are coloured (see key for colours corresponding to domains). Tal - Talitrus saltator, Dp - Daphnia pulex, Ep - Eurydice pulchra, Drom - Drosophila melanogaster, Dplex - Danaus plexippus, Tc - Tribolium castaneum, Ae - Aedes aegypti

\section{PERIOD}

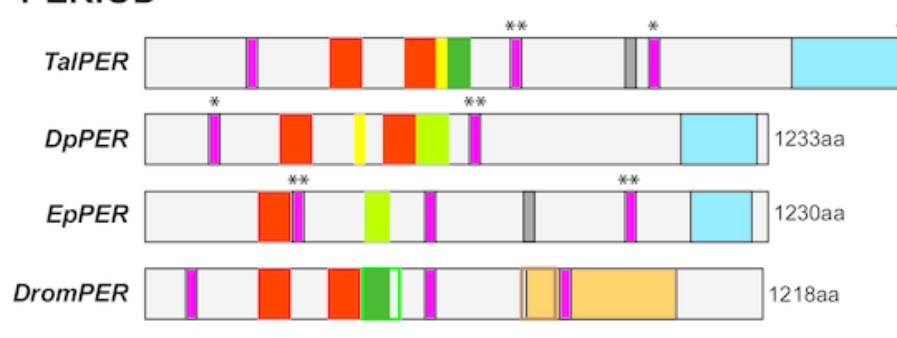

\section{CRYPTOCHROME 2}

\begin{tabular}{|c|c|c|}
\hline TalCRY2 & DNA photolyase & FAD binding 7 \\
\hline EpCRY2 & DNA photolyase & FAD binding 7 \\
\hline
\end{tabular}

\begin{tabular}{|l|l|l|l|} 
DplexCRY2 & DNA photolyase & FAD binding 7 & \\
\hline
\end{tabular}

\begin{tabular}{l||l|l|l|l} 
DromCRY2 & DNA photolyase & & FAD binding 7 & 542aa \\
\cline { 2 - 3 } & &
\end{tabular}

\section{CLOCK}

\section{CYCLE/BMAL1}
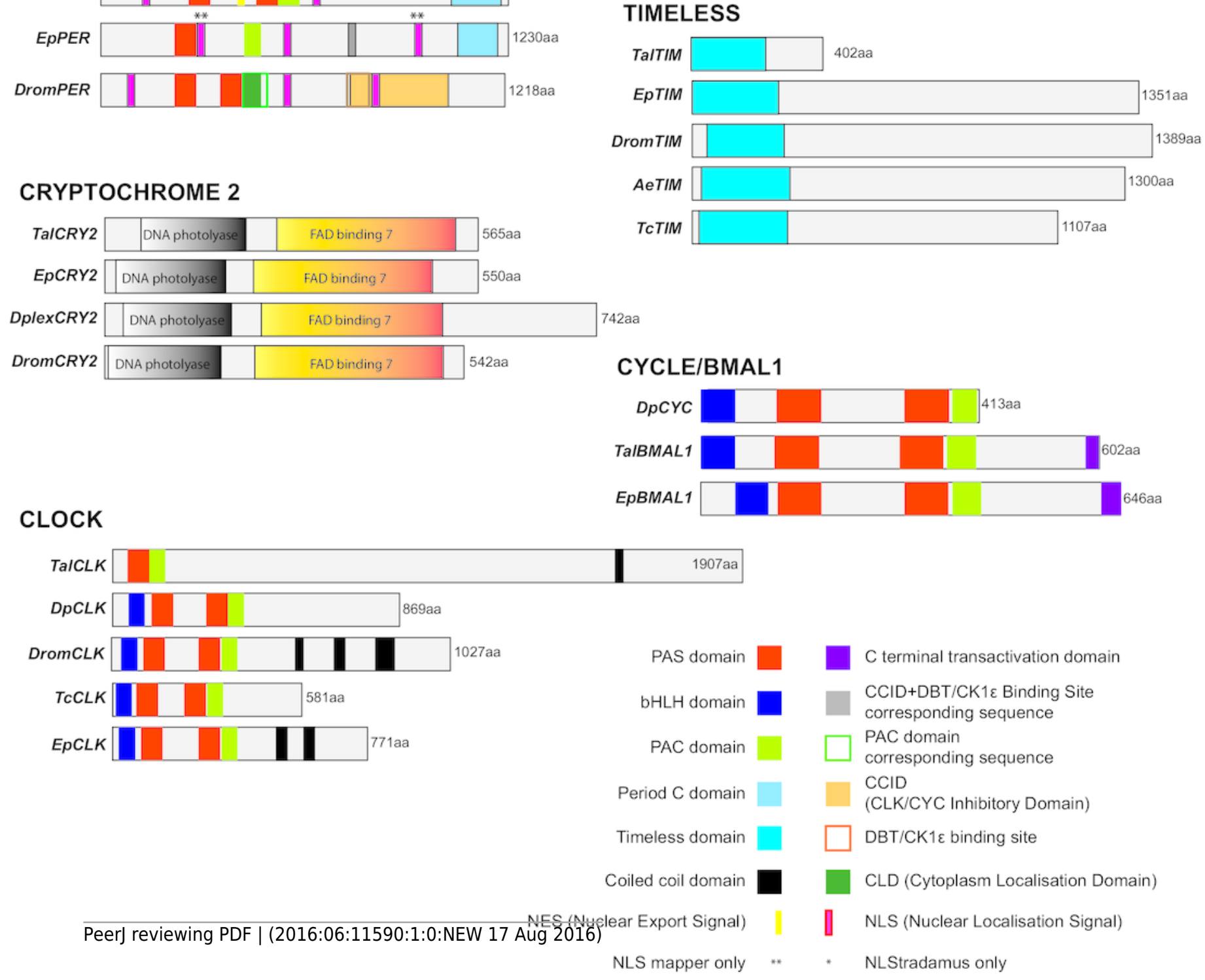


\section{Figure 3 (on next page)}

Talitrus saltator brain tissue transcriptome BLAST2GO analysis

Ontology level 2 data showing functional classification of the brain transcripts from $T$. saltator for three main gene ontology categories 


\section{PeerJ $J^{1 \times}$ Manuscript to be reviewed}
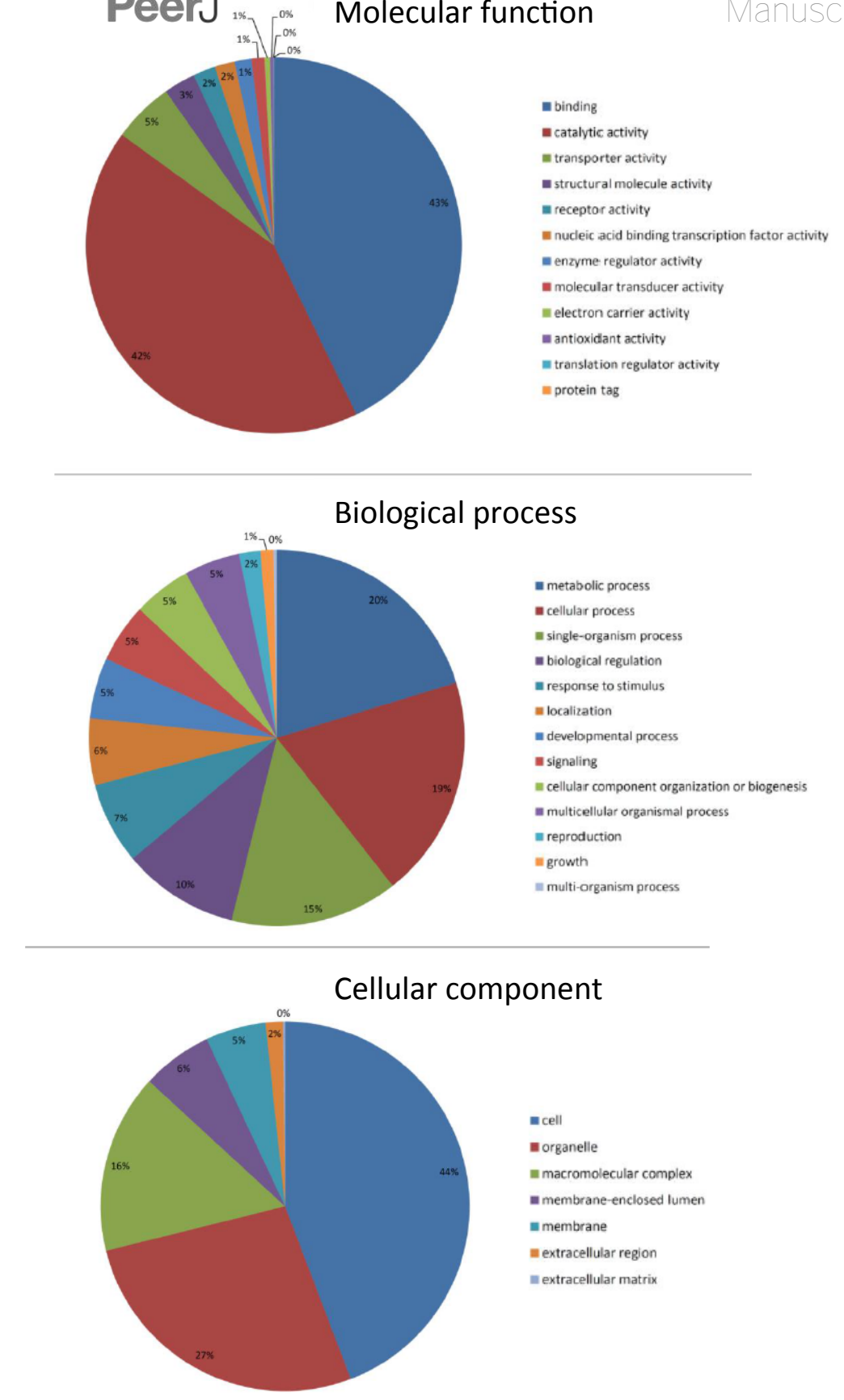

Peer) reviewing PDF | (2016:06:11590:1:0:NEW 17 Aug 2016) 


\section{Figure 4}

Temporal expression profiles of gene transcripts in the brains of free-running Talitrus saltator

Plots show TPM values of each identified core clock and clock-associated genes over a $27 \mathrm{~h}$ period. Data points represent mean TPM values +/- SEM ( $N=4$ biological replicates, except CT18 where $\mathrm{N}=3$ ). Significance values are shown in red for JTK_CYCLE and in blue for ANOVA $\left(\mathrm{F}_{7,30}\right)$. Grey and black bars indicate expected daytime and nighttime, respectively. 

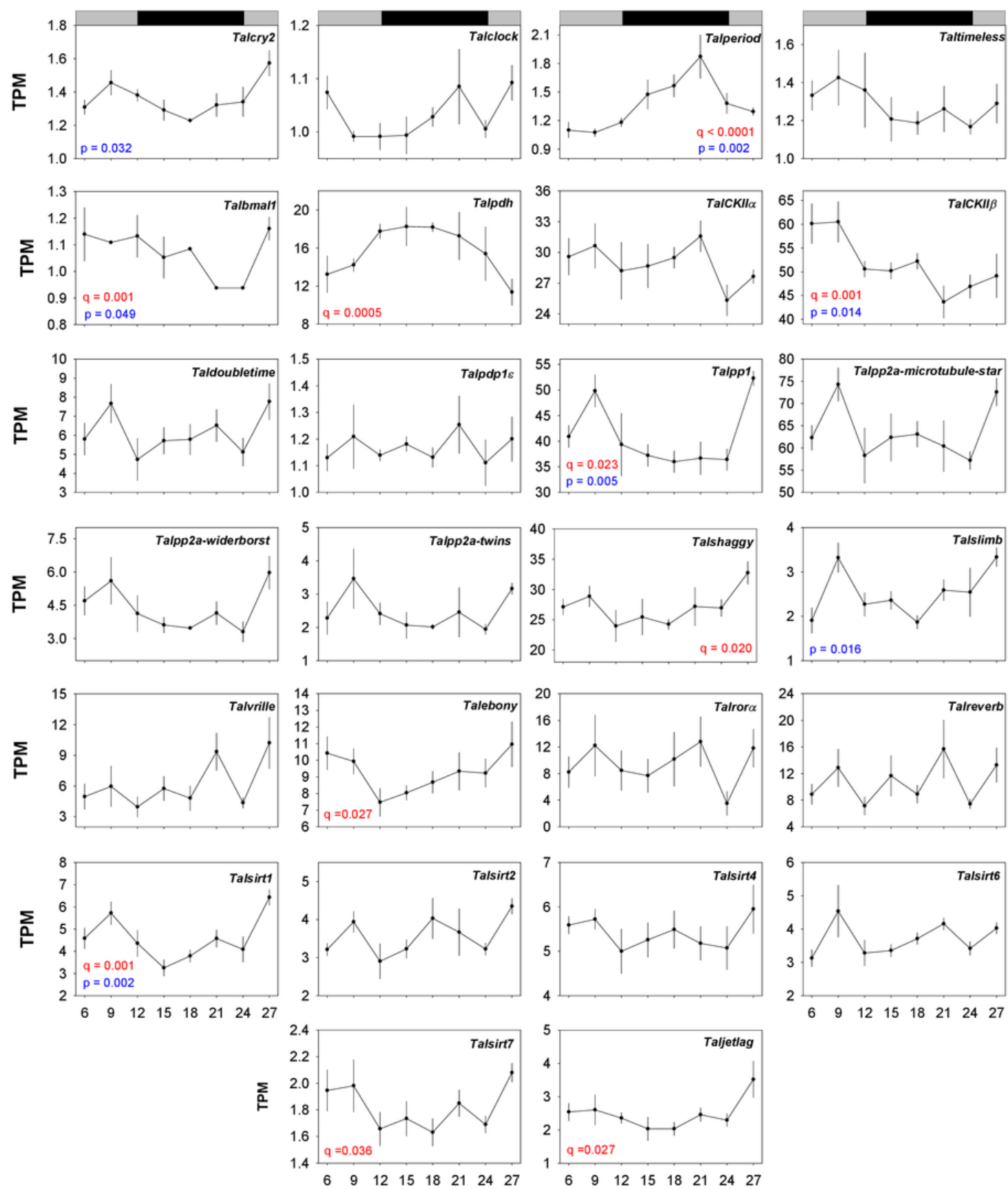

Circadian time (h) 


\section{Table $\mathbf{1}$ (on next page)}

Identified putative Talitrus saltator circadian protein-encoding transcripts

a length in nucleotides. ${ }^{b}$ length in amino acids. ${ }^{c}$ Homology cloning sequence used to search transcriptome. Trinity contigs likely from misassembly, other gene details stated taken from homology cloning and RACE. ' Trinity contig extended 3' sequence past RACE sequence, other gene details stated taken from contig sequence. ${ }^{\text {e }}$ Overlapping contigs combined. ${ }^{f}$ Uca pugilator octadecapeptide $\beta$-PDH consensus sequence used. ${ }^{9}$ Includes combined contig sequence and overlapping 5' RACE sequence. 
1

\begin{tabular}{|c|c|c|c|c|c|c|}
\hline \multirow[t]{3}{*}{ Clock gene query protein } & \multirow{3}{*}{$\begin{array}{l}\text { Transcriptome search } \\
\text { sequence Accession No. } \\
\text { (all D. melanogaster) }\end{array}$} & \multicolumn{5}{|c|}{ Talitrus transcript/protein identifications } \\
\hline & & \multicolumn{3}{|l|}{ Transcript } & \multicolumn{2}{|l|}{ Protein } \\
\hline & & Trinity contig ID & Length $^{\mathrm{a}}$ & RACE & Name & CDS \\
\hline \multicolumn{7}{|l|}{ Core clock proteins } \\
\hline CRY2 & $\mathrm{n} / \mathrm{a}^{\mathrm{c}}$ & $\begin{array}{l}\text { comp100937_c0_seq1 }{ }^{\mathrm{c}} \\
\text { comp102609_c0_seq } 3^{\mathrm{c}}\end{array}$ & 1843 & $5^{\prime}+3^{\prime}$ & Tal-CRY2 & Full \\
\hline CLOCK & AAC62234 & comp100688_c1_seq1 & 5723 & 3 'd & Tal-CLK & Partial \\
\hline PERIOD & AAF45804 & comp102279_c0_seq7 & 8001 & & Tal-PER & Full \\
\hline TIMELESS & AAC46920 & comp849619_c0_seq1 & 1209 & & Tal-TIM & Partial \\
\hline CYCLE & AAF49107 & $\begin{array}{l}\text { comp } 12103 \text { c0_seq } 1 \\
\text { comp939723_c0_seq1 }\end{array}$ & $1807^{\mathrm{e}}$ & & Tal-BMAL1 & Full \\
\hline \multicolumn{7}{|l|}{ Clock-associated proteins } \\
\hline PDH & $\mathrm{n} / \mathrm{a}^{\mathrm{f}}$ & $\begin{array}{l}\text { comp92607_c0_seq2 } \\
\text { comp97165_c0_seq3 }\end{array}$ & $\begin{array}{r}2471^{\mathrm{g}} \\
3392\end{array}$ & $5^{\prime}+3^{\prime d}$ & $\begin{array}{l}\text { Tal-PDH I } \\
\text { Tal-PDH II }\end{array}$ & $\begin{array}{l}\text { Full } \\
\text { Full }\end{array}$ \\
\hline CASEIN KINASE $2 \alpha$ & AAN11415 & comp102480_c0_seq1 & 5147 & & Tal-CK2 $\alpha$ & Full \\
\hline CASEIN KINASE $2 \beta$ & AAF48093 & comp99101_c0_seq3 & 1567 & & Tal-CK2 $\beta$ & Full \\
\hline CLOCKWORK ORANGE & AAF54527 & comp1009591_co_seq1 & 503 & & Tal-CWO & Partial \\
\hline DOUBLETIME (or CK1 $\varepsilon$ ) & AAF57110 & comp87763_co-se- 1 & 1092 & & Tal-DBT & Full \\
\hline PDP1 $\varepsilon$ & AAF04509 & comp98345_c0_seq1 & 3423 & & Tal-PDP1 $\varepsilon$ & Full \\
\hline PP1 & CAA39820 & comp97405_c0_seq1 & 1725 & & Tal-PP1 & Full \\
\hline PP2A - subunit MICROTUBULE STAR & AAF52567 & comp98380_c0_seq1 & 2981 & & Tal-MTS & Full \\
\hline PP2A - subunit WIDERBORST & AAF56720 & 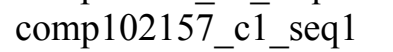 & 2474 & & Tal-WBT & Full \\
\hline PP2A - subunit TWINS & AAF54498 & comp99704_co_seq3 & 1633 & & Tal-TWS & Full \\
\hline SHAGGY & AAN09084 & comp99811_c0_seq7 & 4413 & & Tal-SGG & Full \\
\hline SUPERNUMERARY LIMBS & AAF55853 & comp98870_c0_seq1 & 2121 & & Tal-SLIMB & Full \\
\hline VRILLE & AAF52237 & 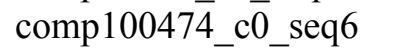 & 3949 & & Tal-VRI & Full \\
\hline EBONY & AAF55870 & comp99283_c0_seq2 & 4380 & & Tal-EBONY & Full \\
\hline RORA & NP_788301 & comp99654_c0_seq3 & 2217 & & Tal-RORA & Partial \\
\hline REVERB & NP_-730321 & 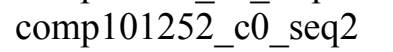 & 5385 & & Tal-REVERB & Full \\
\hline SIRT1 & NP_477351 & comp101818_c1_seq1 & 4033 & & Tal-SIRT1 & Partial \\
\hline SIRT2 & NP_650880 & comp97450_c0_seq4 & 2275 & & Tal-SIRT2 & Partial \\
\hline SIRT4 & NP_572241 & comp92313_c0_seq2 & 2977 & & Tal-SIRT4 & Partial \\
\hline SIRT6 & NP_649990 & comp69157_c0_seq1 & 1209 & & Tal-SIRT6 & Partial \\
\hline SIRT7 & NP_651664 & comp95761_c0_seq1 & 5180 & & Tal-SIRT7 & Partial \\
\hline JETLAG & NP 608880 & comp $10042 \overline{3} \_$c 0 _seq4 & 2454 & & Tal-JET & Partial \\
\hline
\end{tabular}


